\title{
Original
}

\section{A Monte Carlo Study of Photon Beam Characteristics on Various Linear Accelerator Filters}

\author{
Pawiro S. A. ${ }^{1 * \odot}$, Azzi A. ${ }^{2}$, Soejoko D. S. ${ }^{1}$
}

\begin{abstract}
Background: Intensity Modulated Radiation Therapy (IMRT) technique is an advanced method of radiotherapy leading into the development of Flattening FilterFree (FFF) medical linear accelerators (Linacs). Monte Carlo simulation has been a standard method for calculation of particle transport due to precise geometry and material specifications.

Objective: This study is to obtain the design optimization of Flattening Filter Free (FFF) for 6 MV Linac machine.

Material and Methods: In this simulating study, EGSnrc user code was used to simulate particles emitted from head of linac 6MV Varian to achieve the most suitable filter in FFF linac design. Monte Carlo simulation results of the PDD and profile, on the $10 \times 10 \mathrm{~cm}^{2}$ field, were compared with the measurements. Differences in small profile beams from Monte Carlo simulation were also evaluated between FF and FFF linac.
\end{abstract}

Results: The spectrum on Monte Carlo simulation in isocenter was compared with Treatment Planning System (TPS) for each filter variation. The slight differences of average spectrum are simulated using $2 \mathrm{~mm}$ copper filter and FakeBeam with $-1.52 \pm 3.82 \%$ and $-3.13 \pm 3.61 \%$. Whereas, for PDD and profiles, each variation has an average difference of $7.10 \pm 0.70 \%$ and $-5.99 \pm 1.39 \%$.

Conclusion: FakeBeam filter is a proper filter for the use of linac design 6MV Varian. It is necessary to decrease the kinetic energy of electrons to perform MC simulations on FFF linac.

Citation: Pawiro SA, Azzi A, Soejoko DS. A Monte Carlo Study of Photon Beam Characteristics on Various Linear Accelerator Filters. $J$ Biomed Phys Eng. 2020;10(5):613-622. doi: 10.31661/jbpe.v0i0.1192.

\section{Keywords}

FFF Linac; Filter Design; Radiotherapy; Particle Accelerators; Radiation

\section{Introduction}

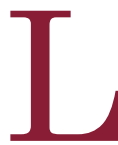
inear accelerator (linac) is a proper machine to accelerate particles and reach a certain high kinetic energy. Recently, linac, with high-energy electron or bremsstrahlung X-ray, has been implemented in clinics as one facility to improve cancer treatments. Bremsstrahlung X-ray produced by linac has a non-uniform distribution so that a filter called flattening filter (FF) is used to obtain a homogeneous energy fluence on a large radiation field in the patient, generally up to $40 \times 40 \mathrm{~cm}^{2}[1]$.

A modern linac has commonly implemented intensity modulated ra-
${ }^{1} \mathrm{PhD}$, Department of Physics, Faculty of Mathematics and Natural Sciences, University of Indonesia, Depok, Indonesia

${ }^{2} \mathrm{MSc}$, Department of

Physics, Faculty of Math-

ematics and Natural

Sciences, University

of Indonesia, Depok,

Indonesia

*Corresponding author: S. A. Pawiro

Department of Physics, Faculty of Mathematics and Natural Sciences,

University of Indonesia,

Depok, Indonesia

E-mail: supriyanto.p@sci. ui.ac.id

Received: 31 May 2019 Accepted: 15 July 2019 
diation therapy (IMRT) technique in order to achieve better precision and accuracy on the cancer treatment irradiation. IMRT has applied jaws and multi-leaf collimators (MLCs) to shape photon distribution referring to the target volume. MLC has been also used to modulate the energy fluence inside the tumor volume. This modulation builds a non-uniform dose volume at the planning target volume to support organs at risk nearby. As a result, the flat beam is not necessary on IMRT irradiation technique and the flattening filter can be removed. The linac without the flattening filter is called flattening filter free (FFF) [2].

Monte Carlo (MC) has been a standard method for calculation of particle transport and widely used to simulate high energy X-ray beams in linac with flattening filters. The FF linac head specification can be found on the Monte Carlo data package [3]. On the other hand, the flattening filter is replaced with other filter material to maintain the signal received by monitor chambers on FFF linac design [4]; however, the filter design is not provided by manufacturers. Thus, many researchers have been predicted and simulated design of the FFF linac head especially on the flattening filter replacement material [5-7]. Several studies have been conducted to measure and compare 6MV photon beam characteristics, both on Monte Carlo simulations and measurements. Differences in the characteristics of photon beams are usually evaluated as percentage depth dose (PDD), maximum depth, beam profile, penumbra, output factor (OF), energy spectrum, surface dose, beam quality, and the alignment of photon [6-12]. This research aims to design a proper filter for the best flattening filters using Monte Carlo simulations, especially for small field irradiation techniques. We evaluated the spectrum ratio of FF and FFF linac using 4 filter variations. Furthermore, comparison of beam air profile for field sizes, including $1 \times 1,2 \times 2,3 \times 3$, and $4 \times 4 \mathrm{~cm}^{2}$ was performed using Monte Carlo simulation between FF and FFF linac.

\section{Material and Methods}

This experimental and simulation studies were carried out using linac 6MV photon beam of Varian Trilogy, which was modified to operate on FFF photon beam at Pasar Minggu Regional General Hospital. The measurement was performed using a water tank phantom and ionization chamber detector type CC13. Besides, we employed EGSnrc user code to simulate the particles transport that implemented Monte Carlo method.

\section{Measurement and TPS Calculation}

We used Blue Phantom2 (iBa dosimetry), which had a field $48 \times 48 \times 48 \mathrm{~cm}^{3}$ in volume to perform our measurement. A pair of ionization chamber detectors named Compact Chamber 13 (CC13) with an active volume of $0.13 \mathrm{cc}$ was set for relative dose measurement coupled to MyQAccept application software in order to control the movement of the detector. The detector was remotely moved by continuous scan protocol with a constant speed of $1.5 \mathrm{~mm} / \mathrm{s}$. The phantom was irradiated with field size of $10 \times 10 \mathrm{~cm}^{2}$ on the surface of the phantom and the source to surface distance (SSD) of $100 \mathrm{~cm}$. We focused on the characteristics of PDD and profile at $10 \mathrm{~cm}$ depth of the water phantom.

Eclipse v.13, which has an analytical anisotropic algorithm (AAA), was used to calculate the interest beam characteristics as the calculation of treatment planning system (TPS). The beam data commissioning for this calculation was imported from the several measurement results using the same ionization detectors. Furthermore, the photon spectrum of FFF linac 6MV was extracted from TPS beam for the comparison purpose to the Monte Carlo simulation. The spectrum of TPS photon beam was set with no material filter.

Monte Carlo simulation parameters

The head of FF linac design obtained from Varian Monte Carlo data package contained 8 geometry structures, including target, pri- 
mary collimator, vacuum glass, flattening filter, monitor chamber, mirror, jaws, and air until SSD $100 \mathrm{~cm}$. Whereas, FFF linac design was constructed from the same design as FF LINAC with a modification at flattening filter material and geometry. We used 4 replacement filters, such as FakeBeam design with $0.8 \mathrm{~mm}$ of brass, $2 \mathrm{~mm}$ of copper, and without filter (literally no filter attached) [4-7].

Since the real geometry and material were designed for FF linac, we did trial and error to obtain the electron source kinetic energy and full width half-maximum (FWHM) using the FF linac design and then implemented the electron source to all FFF linac design. This was performed for the kinetic energy and FWHM from 5.8 to $7.2 \mathrm{MeV}$ and 0.10 to $0.20 \mathrm{~cm}$, respectively. Particle history was $10^{8}$ particles with photon cut-off energy (PCUT) of 0.01 $\mathrm{MeV}$ and electron cut-off energy (ECUT) of $0.70 \mathrm{MeV}$ included its rest mass. We used direct bremsstrahlung split (DBS) method with a radius of $10 \mathrm{~cm}$ to increase the efficiency of simulation time and the particle direction to $\mathrm{Z}$ positive or straightforward to the head linac. Phase space files were taken at the end of the air material which meant $100 \mathrm{~cm}$ of SSD to examine energy spectrum and fluence laterally for field sizes, including $1 \times 1,2 \times 2,3 \times 3$ and $4 \times 4 \mathrm{~cm}^{2}$. Moreover, phase space file of $10 \times 10$ $\mathrm{cm}^{2}$ was practically used as a particle source on a water phantom using the DOSXYZnrc user code to evaluate the percentage depth dose (PDD) and the beam profile at a depth of $10 \mathrm{~cm}$. The DOSXYZnrc result of FFF linac was then compared to the MC FF linac and the measurement of FFF linac. Besides, we used BEAMDP user code in which one of the add-ons in EGSnrc was to obtain the spectrum and the beam air profile for small field evaluation. The water phantom of DOSXYZnrc was built by a voxel size of $0.25 \times 0.25 \times 0.25 \mathrm{~cm}^{3}$, whereas the beam air profile was generated by $0.025 \mathrm{~cm}$ spatial resolution laterally.

Dose evaluation between FF and FFF linac was performed by comparing a relative dose at specific points called local dose comparison following Equation (1):

$$
\% D=\frac{\left(D_{s}-D_{p}\right)}{D_{s}} \times 100 \%
$$

Where $\% D$ is local dose comparison, $D_{s}$ and $D_{p}$ are relative doses obtained by simulation and measurement, respectively.

\section{Results}

Initial electron kinetic energy and FWHM, which were $7.0 \mathrm{MeV}$ and $0.2 \mathrm{~cm}$, respectively, for $\mathrm{FF}$ linac led into the best match for local dose comparison between MC and measurement on PDD and profile of $10 \times 10 \mathrm{~cm}^{2}$ photon beam. Furthermore, these parameters were used for the electron source of FFF linac design.

\section{FF and FFF linac energy spectrum}

The ratio of FF and FFF linac photon spectrum with various filters using the Monte Carlo simulation are displayed in Figure 1. The energy ratio in Figure 1 is just displayed until 1 $\mathrm{MeV}$ because the energy, thereafter, has a ratio by a relatively constant value. The highest ratio was shown without filter design, while the ratio of $2 \mathrm{~mm}$ copper and Fakebeam showed better results. The results show that $2 \mathrm{~mm}$ copper filter has a considerable thickness with the maximum ratio of the spectrum between FF and FFF linac of 3.22 .

Figure 2 shows the comparison of photon energy spectrum for FFF linac between MC and TPS beam based on percent normalized at $0.375 \mathrm{MeV}$ for all filters. It obviously shows that spectrum of $2 \mathrm{~mm}$ copper filter and Fakebeam intercepted the zero line ratio at some points, whereas $0.8 \mathrm{~mm}$ brass filter and without filter were far from spectrum calculated by TPS.

\section{PDD and profile at $10 \times 10 \mathrm{~cm}^{2}$}

The comparison of PDD and profile on the radiation field, which is $10 \times 10 \mathrm{~cm}^{2}$, between $\mathrm{MC}$ simulation and measurements using ionization 
Pawiro S. A., Azzi A., Soejoko D. S.

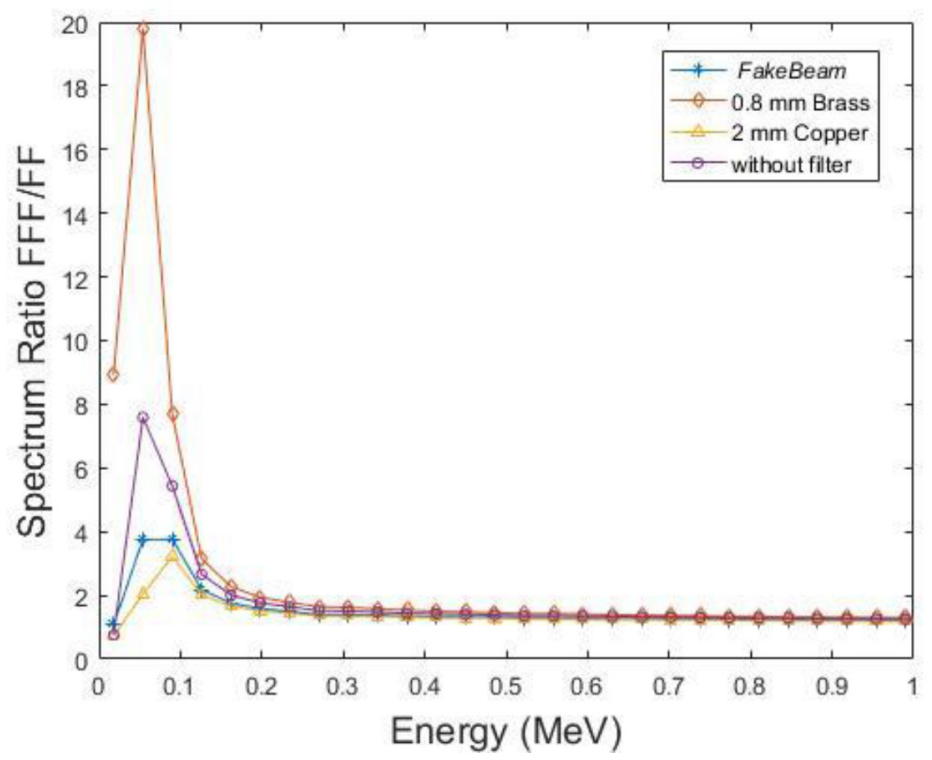

Figure 1: Spectrum ratio of flattening filter (FF) and flattening filter free (FFF) photon beams for FakeBeam, $0.8 \mathrm{~mm}$ brass, $2 \mathrm{~mm}$ copper, and without filter. The energy was just displayed until $1 \mathrm{MeV}$ because the following energy ratio was 1.

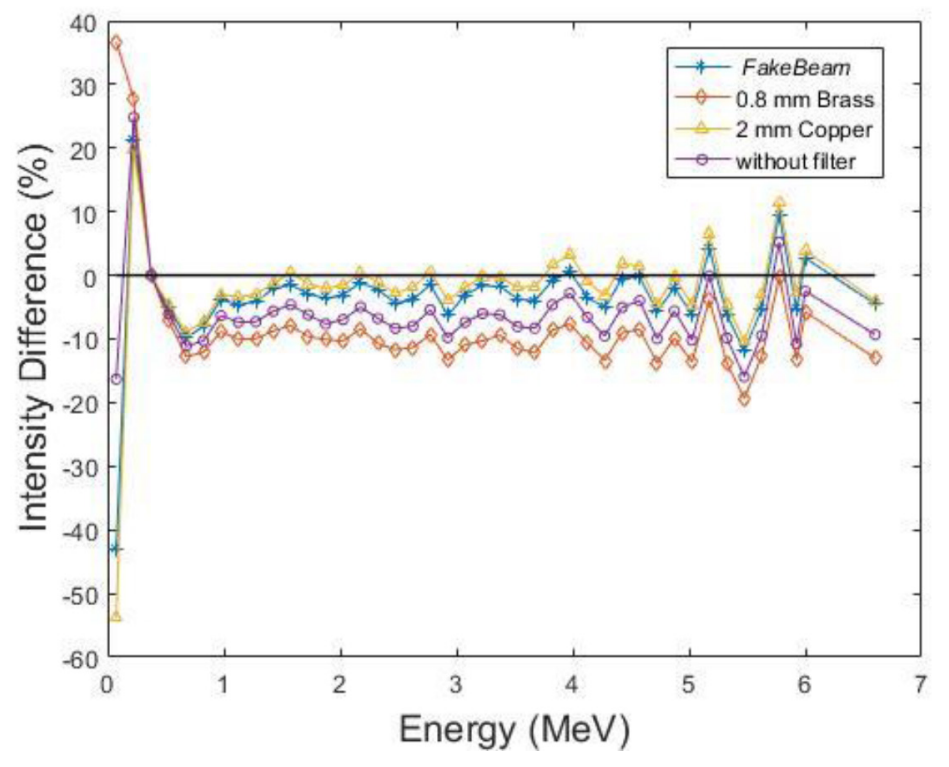

Figure 2: Intensity difference of photon energy spectrum 6MV flattening filter (FF) and flattening filter free (FFF) beam for FakeBeam, $0.8 \mathrm{~mm}$ brass, $2 \mathrm{~mm}$ copper, and without filter.

chamber detector was performed. Evaluation of PDD was carried out along with a center of axis (CAX) from the water phantom surface until $30 \mathrm{~cm}$ depth. The results of the local dose comparison between MC FF and FFF linac simulation can be seen in Figure 3. The data shows that the lowest dose difference between $\mathrm{FF}$ and FFF linac was at $0.8 \mathrm{~mm}$ brass filter followed by $2 \mathrm{~mm}$ copper, Fakebeam, and without filter. The average deviations of the PDDs excluding the build-up area were -4.27 $\pm 1.97 \%,-3.73 \pm 1.74 \%,-4.28 \pm 1.88 \%$, and 
Beam Characteristics on Various Filter

$-4.80 \pm 1.8 \%$ for Fakebeam, $0.8 \mathrm{~mm}$ brass, 2 $\mathrm{mm}$ copper, and without filter, respectively.

On the order hand, the average dose difference of the PDD including the build-up region of MC simulation compared to measurement for Fakebeam, $0.8 \mathrm{~mm}$ brass, $2 \mathrm{~mm}$ copper, and without filter were $6.20 \pm 5.12 \% ; 9.72 \pm$
$7.55 \% ; 3.63 \pm 2.69 \%$; and $2.78 \pm 3.98 \%$, respectively. However, the difference of PDDs tends to positively increase with a deeper point of interest between $\mathrm{MC}$ and measurement of FFF linac as shown in Figure 4.

Furthermore, we evaluated the beam profile of FFF linac which considered the non-flat

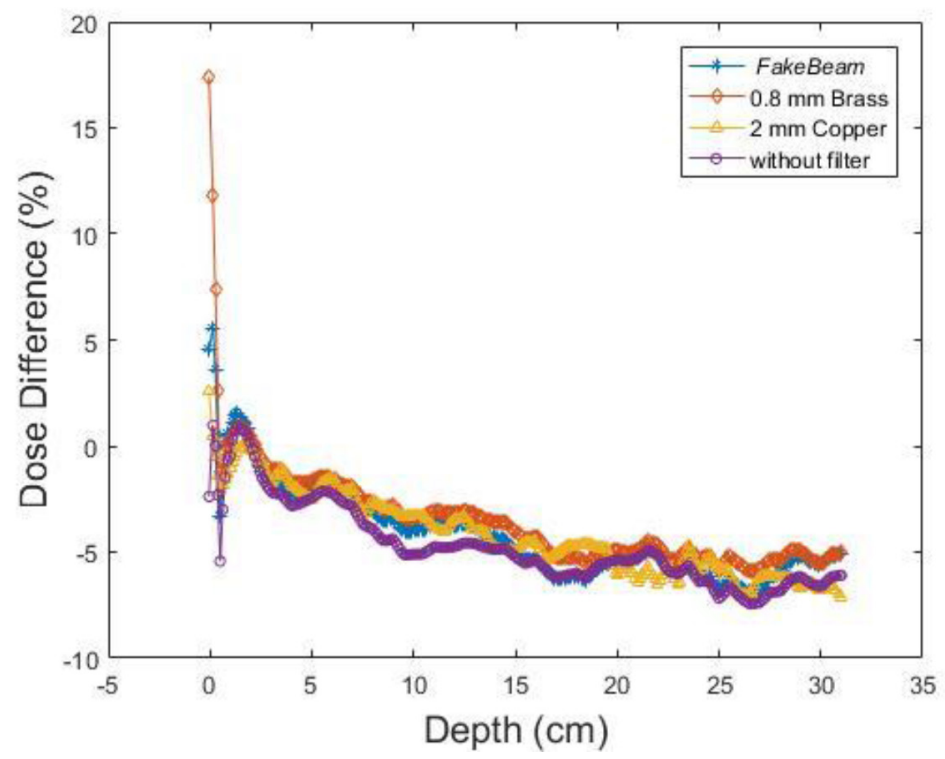

Figure 3: Difference of percentage depth dose between simulated 6MV flattening filter (FF) and flattening filter free (FFF) photon beams for FakeBeam, $0.8 \mathrm{~mm}$ brass, $2 \mathrm{~mm}$ copper, and without filter.

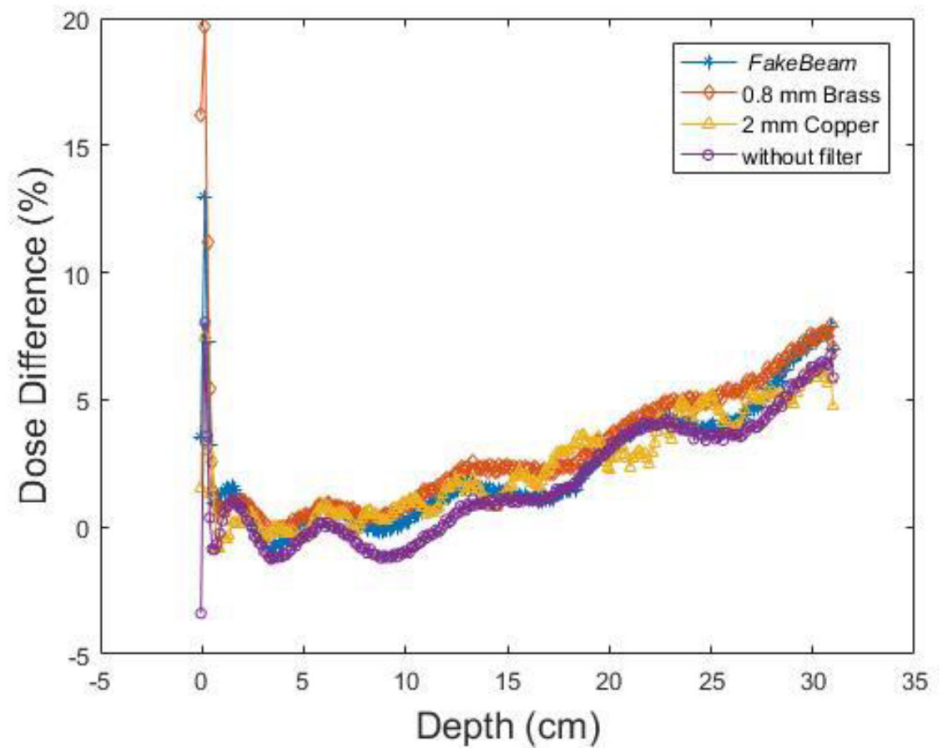

Figure 4: Percentage of depth dose difference between simulated 6MV flattening filter free (FFF) and measured FFF photon beams for FakeBeam, $0.8 \mathrm{~mm}$ brass, $2 \mathrm{~mm}$ copper, and without filter. 
beam for the replacement of a flattening filter. Local dose difference of MC simulation between FF and FFF linac can be seen in Figure 5. The results described the softened of the beam profile of FFF linac in which the dose difference was 0 at CAX and tended to $10 \%$ at the edge of the radiation field size. Moreover, the dose difference at off-axis after $7 \mathrm{~cm}$ from CAX shows that negative value, around $10 \%$, led into the lower dose for FFF than FF. Visually, there was no significant dose difference of lateral beam profile from both of variation of filters. The averages of dose differences, which were quantitatively calculated along 10 $\mathrm{cm}$, were $-4.13 \pm 2.90 \%,-4.49 \pm 3.67 \%,-5.55$ $\pm 3.91 \%$, and $-5.24 \pm 3.73 \%$ for Fakebeam, $0.8 \mathrm{~mm}$ brass, $2 \mathrm{~mm}$ copper, and without filter, respectively.

On the order hand, the dose difference of FFF linac between $\mathrm{MC}$ simulation and measurement can be seen in Figure 6. We achieved that the averages of dose difference for Fakebeam, $0.8 \mathrm{~mm}$ brass, $2 \mathrm{~mm}$ copper, and without filter were $-0.35 \pm 1.21 \%,-0.76 \pm 1.32 \%,-1.85 \pm$ $1.75 \%$, and $-1.54 \pm 1.49 \%$, respectively.

\section{Beam profile in small field sizes}

We evaluated the outcome of MC energy flu- ence of field sizes, including $1 \times 1,2 \times 2,3 \times 3$, and $4 \times 4 \mathrm{~cm}^{2}$ between FF and FFF linac. The analysis of beam air profile was conducted to obtain the characteristics, which were different from the small field on our various filters to flattening filter as shown in Figure 7. It shows that all of the various FFF filters indicated the same relative dose. The deviation of air beam profile affected by the flattening filter was visually seen on the periphery of the beam at $3 \times 3$ and $4 \times 4 \mathrm{~cm}^{2}$. Meanwhile, field sizes such as $1 \times 1$, and $2 \times 2 \mathrm{~cm}^{2}$ between FF and FFF photon beams were relatively identical both of visual and quantity along with the irradiation beam size. These results show that lateral dose from the center axis up to the beam edge of FF and FFF was similar for the field size of $2 \times 2 \mathrm{~cm}^{2}$ or smaller. However, there was a significant dose difference at the out of beam field size as seen in Figure 8. According to the results, the variation of FFF filter design did not have any impact on relative dose profile in the small field area.

\section{Discussion}

This study investigated the effect of flattening filter on the dose distribution of photon particles using Monte Carlo method. The sim-

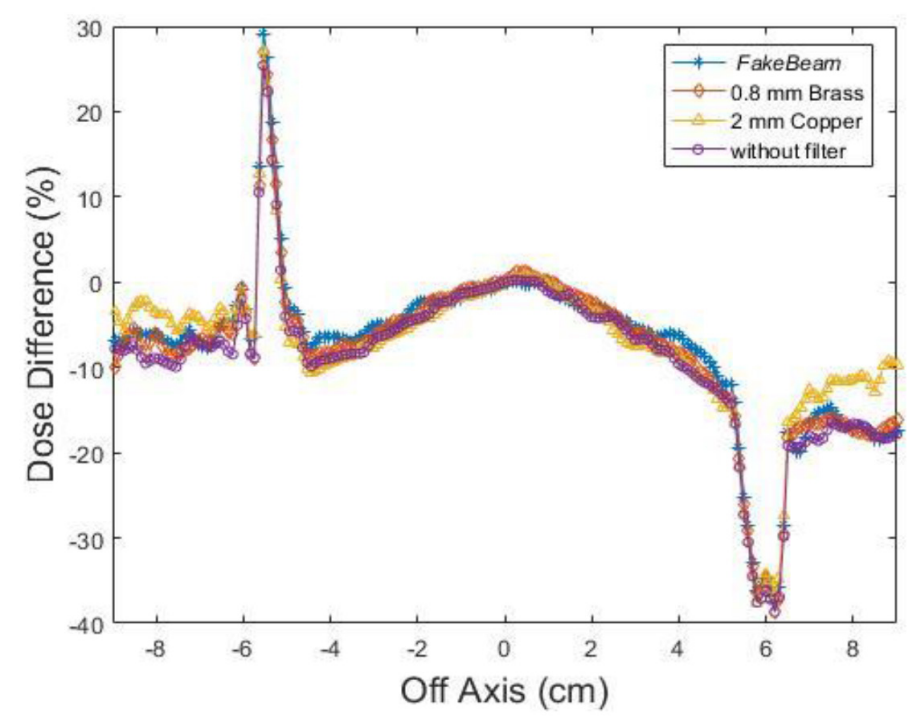

Figure 5: Percentage of dose difference of cross lane off axis between simulated 6MV flattening filter (FF) and flattening filter free (FFF) photon beams for FakeBeam, $0.8 \mathrm{~mm}$ brass, $2 \mathrm{~mm}$ copper, and without filter. 


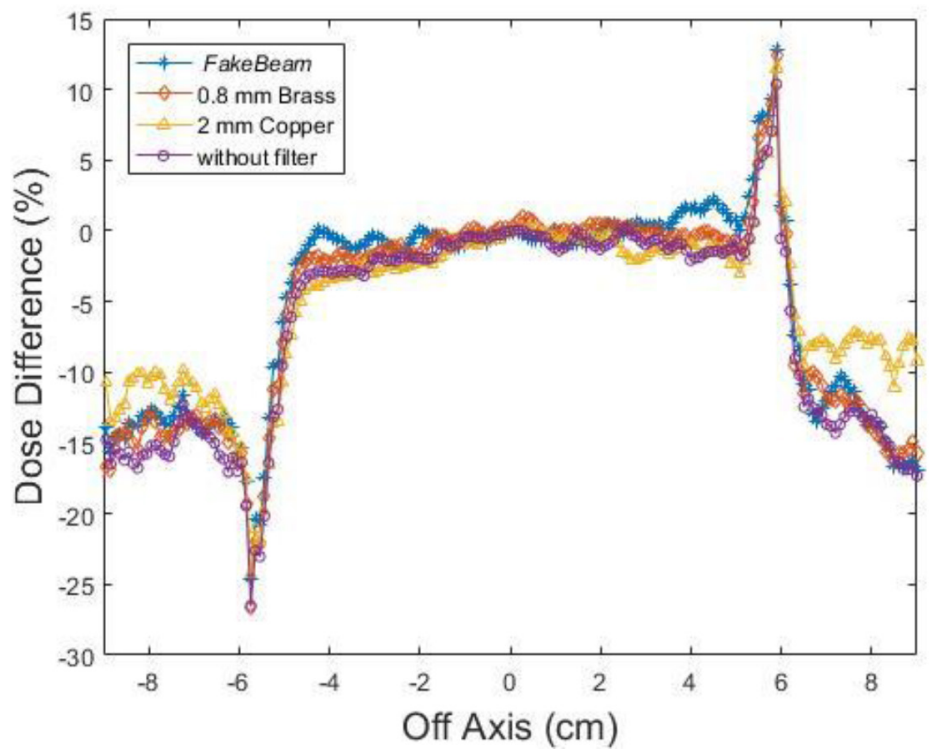

Figure 6: Percentage of dose difference of cross lane off axis between simulated 6MV flattening filter (FF) and flattening filter free (FFF) photon beams for FakeBeam, $0.8 \mathrm{~mm}$ brass, $2 \mathrm{~mm}$ copper, and without filter.

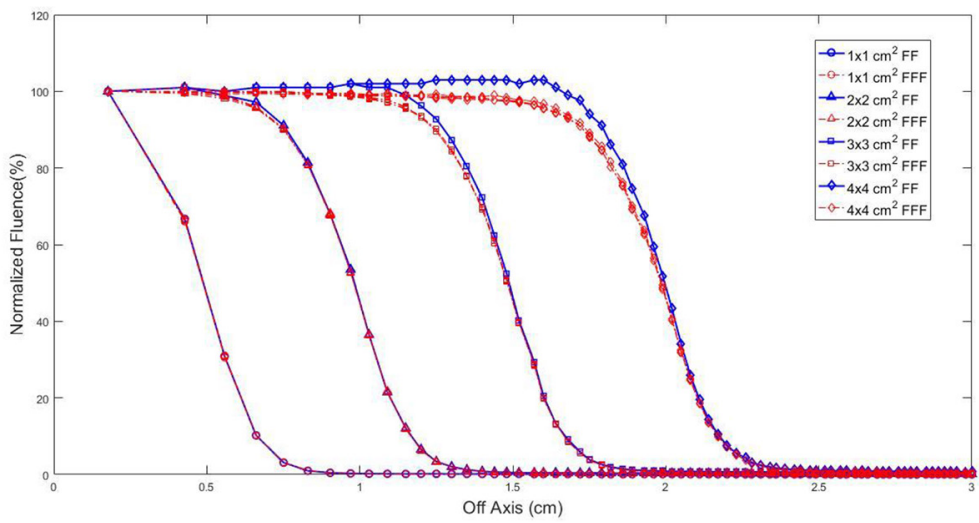

Figure 7: Fluence percentage of air profile for flattening filter (FF) and flattening filter free (FFF) linac for small field sizes

ulation was conducted based on five difference structures and materials of flattening filter, including regular 6MV linac, FakeBeam, $0.8 \mathrm{~mm}$ brass, $2 \mathrm{~mm}$ copper, and without filter. Regular 6MV linac flattening filter is considered to be a standard of FF linac design in which the Monte Carlo simulation was calibrated to the measurement on this filter design. Another filter was for FFF linac because the blueprint of FFF linac is a confidential file. In this study, we evaluated the purposed filters based on the Monte Carlo simulation for photon spectrum,
PDD and profile of filed sizes $10 \times 10 \mathrm{~cm}^{2}$ and smaller for photon beams.

Energy spectrum output of 6MV FF and FFF linac was different due to the interaction of photon and other particle contaminants such as electron and positron with the material and flattening filter. Different ratio was computed between FF and FFF linac, which seemed high on the low energy spectrum. This effect was found because the absence of beam hardening in flattening filter on the FFF linac head design. On the beam of TPS eclipse-based, we 


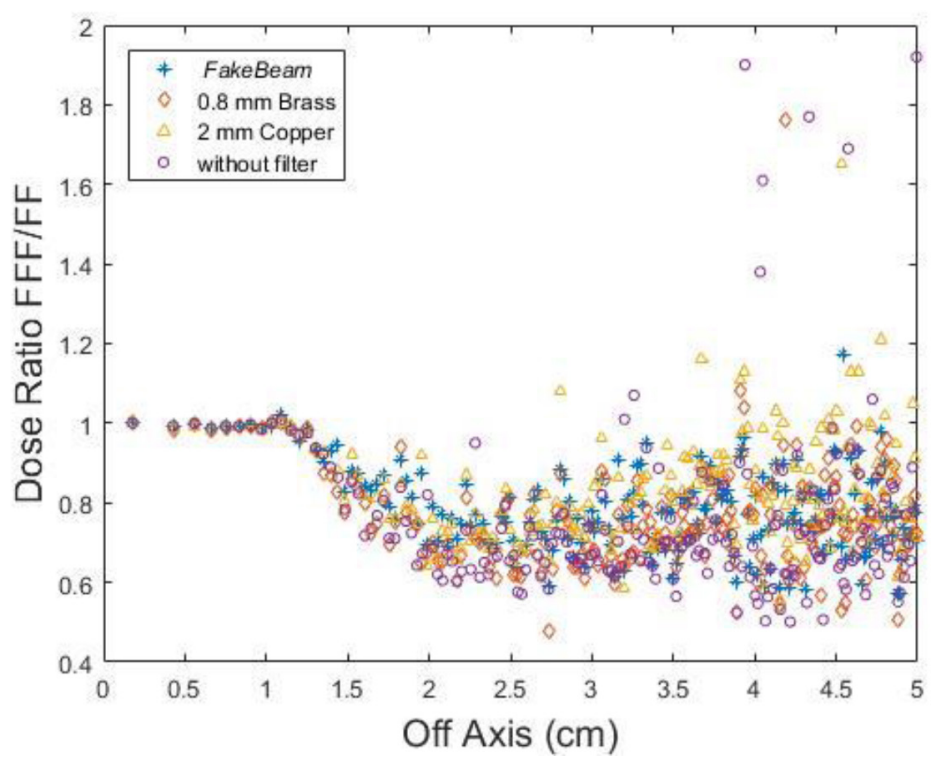

Figure 8: Dose ratio of flattening filter free (FFF) by flattening filter (FF) photon beam at $2 \times 2 \mathrm{~cm}^{2}$ field sizes for FakeBeam, $0.8 \mathrm{~mm}$ brass, $2 \mathrm{~mm}$ copper, and without filter

extracted the spectrum component and compared the energy spectrum of FFF linac on MC simulation and TPS. The average of spectrum FFF was different between MC simulation and TPS i.e. $-3.13 \pm 3.61 \%,-10.35 \pm 3.12 \%,-1.52$ $\pm 3.82 \%$, and $-6.92 \pm 3.41 \%$ for FakeBeam, $0.8 \mathrm{~mm}$ brass, $2 \mathrm{~mm}$ copper, and without filter, respectively. The best photon spectrum matching to TPS was copper filter of $2 \mathrm{~mm}$ thickness. This showed that although the thickness of material filter was great enough for a beam hardening, which was as much as FF linac, adding $2 \mathrm{~mm}$ thickness into copper filter in flattening filter did not change the characteristic of energy spectrum of FFF linac compared to TPS. Moreover, there was a significant percentage of spectrum difference between MC simulation and TPS at low energy spectrum. We found that the photon spectrum's peak of MC was shifted to lower energy than TPS. The nominal spectrum shift of MC simulation compared to TPS calculation for FakeBeam, $0.8 \mathrm{~mm}$ brass, $2 \mathrm{~mm}$ copper, and without filter were $0.105,0.105,0.069$, and $0.105 \mathrm{MeV}$, respectively.

In a standard field, we did a simulation on
$10 \times 10 \mathrm{~cm}^{2}$ for PDD and profile at $10 \mathrm{~cm}$ depth of water. The trend of PDD difference between FF and FFF linac gradually decreased at great depth. Moreover, the PDD difference between FFF simulation and measurement led to increase at greater depth. It seemed that the initial electron kinetic energy of the MC simulation of FFF linac had to reach an independent relative dose difference in depth for these filters. The better match of MC PDD to measurement in this study was without filter followed by $2 \mathrm{~mm}$ copper, FakeBeam, and $0.8 \mathrm{~mm}$ brass. Besides, we evaluated the dose differences in profile of $10 \times 10 \mathrm{~cm}^{2}$ at $10 \mathrm{~cm}$ depth. An interesting area was found around 5 to $7 \mathrm{~cm}$ from the CAX. In this area, the dose difference between FF and FFF was high. This phenomenon was indicated by beam horns on FF linac; however, the beam horn was not recorded at all FFF linac designs. Furthermore, the FWHM between FFF and FF beam profile at $10 \mathrm{~cm}$ depth was also found different. We investigated that there was a $2 \mathrm{~mm}$ discrepancy on penumbra region between FF and FFF photon beam profile. Based on the IAEA TRS 430 [13], the evaluation of beam profile 
below the half maximum could be conducted by dose difference or deviation of distance of two profiles which had tolerance of $10 \%$ and $2 \mathrm{~mm}$, respectively. Therefore, the sharp area of dose difference was agreed according to the deviation of distance. However, the evaluation of beam umbra i.e. $20 \%$ of maximum beam and lower or outside beam edge only could be carried out based on dose difference parameter with the tolerance maximum of $3 \%$. The dose differences computed between FF and FFF linac on this area were about $-10 \%$ meaning that FFF photon beam had lower dose than FF linac. On the other hand, this result shows that FFF beam was superior in order to reduce dose received at the surrounding organ than FF. Based on the result, FakeBeam design had the better characteristics representation of linac FFF beam profile because the FakeBeam geometry has a special shape rather than other filter design, whereas the largest discrepancy was found at $2 \mathrm{~mm}$ copper filter. As a result, the beam hardening effect was highly produced at off-axis of $2 \mathrm{~mm}$ copper filter.

Moreover, we checked the profile on the small field sizes, including $1 \times 1$ to $4 \times 4 \mathrm{~cm}^{2}$ on photon air profile. The dose profile of FFF actually had the same trend and slight difference with each other. It seems that characteristics of small field were not dependent on structure or material filter. However, if the normalization was not implemented, there would be a different fluence ratio of 1.07, 1.57, 1.45, and 1.65 for FakeBeam, $0.8 \mathrm{~mm}$ brass, $2 \mathrm{~mm}$ copper, and without filter to FF linac because of the different interaction of particles with filter materials. On the other hand, the differences of photon profile in air between FF and FFF linac showed the differences on beam fringe from $90 \%$ until $50 \%$ of maximum dose, especially at quite large field size. Quantitatively, the percentage of discrepancies between all various FFF filters and FF of this area were $3.24 \pm 0.45$ and $6.79 \pm 1.01 \%$ for $3 \times 3$, and $4 \times 4$ $\mathrm{cm}^{2}$, respectively. It indicates that the softened beam as an impact of removing flattening filter was found at larger field size of $2 \times 2 \mathrm{~cm}^{2}$. Furthermore, the dose reduction of the FFF beam was also made at small field sizes as shown in Figure 8. It shows that the dose of FFF photon beam decreased by 0.6 smaller than FF beam at out of field which spared the dose of surround the target volume. According to the dose distribution outside of field, the FakeBeam design had a constant distribution rather than all filters.

\section{Conclusion}

In this study, the best corresponding filter that matches the measurement of FFF linac was Fakebeam design filter. Fakebeam filter was superior in beam lateral of $10 \times 10 \mathrm{~cm}^{2}$ and consistent with PDD beam evaluation. In the small field irradiation, various filters did not differ from each other for beam air profile. The positive local dose difference between FF and FFF indicated that it is necessary to modify the initial energy kinetic to simulate MC head linac of FFF from FF linac design. FFF linac beam had a better dose sparing for organs at risk than FF; however, a comprehensive study for out of field effect of FFF linac is needed.

\section{Acknowledgment}

This study was supported by Pasar Minggu Regional General Hospital and the authors appreciated the help from Dea Ryangga for technical assistance and valuable medical expertise. This study was supported by Universitas Indonesia Research Grant (PIT9) with contact number NKB-0034/UN2.R3.1/ HKP.05.00/2019.

\section{Conflict of Interest}

None

\section{References}

1. Pichandi A, Ganesh KM, Jerin A, Balaji K, Kilara G. Analysis of physical parameters and determination of inflection point for Flattening Filter Free beams in medical linear accelerator. Rep Pract Oncol Radiother. 2014;19:322-31. doi: 10.1016/j. rpor.2014.01.004. PubMed PMID: 25184057. 
Pawiro S. A., Azzi A., Soejoko D. S.

PubMed PMCID: PMCPMC4150098.

2. Ponisch F, Titt U, Vassiliev ON, Kry SF, Mohan R. Properties of unflattened photon beams shaped by a multileaf collimator. Med Phys. 2006;33:173846. doi: 10.1118/1.2201149. PubMed PMID: 16872081 .

3. Anonymous. Monte Carlo data package for High Energy Accelerator: document number 100040466. USA: Varian Medical System; 2003.

4. Xiao Y, Kry SF, Popple R, Yorke E, Papanikolaou $\mathrm{N}$, Stathakis $\mathrm{S}$, et al. Flattening filter-free accelerators: a report from the AAPM Therapy Emerging Technology Assessment Work Group. J App/ Clin Med Phys. 2015;16:5219. doi: 10.1120/jacmp. v16i3.5219. PubMed PMID: 26103482. PubMed PMCID: PMCPMC5690108.

5. Rodriguez M, Sempau J, Fogliata A, Cozzi L, Sauerwein W, Brualla L. A geometrical model for the Monte Carlo simulation of the TrueBeam linac. Phys Med Biol. 2015;60:N219-29. doi: 10.1088/00319155/60/11/N219. PubMed PMID: 25984796.

6. Tsiamas P, Sajo E, Cifter F, Theodorou K, Kappas $\mathrm{C}$, Makrigiorgos $\mathrm{M}$, et al. Beam quality and dose perturbation of $6 \mathrm{MV}$ flattening-filter-free linac. Phys Med. 2014;30:47-56. doi: 10.1016/j. ejmp.2013.02.004. PubMed PMID: 23517668.

7. Sangeetha S, Sureka C. Comparison of Flattening Filter (FF) and Flattening-Filter-Free (FFF) $6 \mathrm{MV}$ photon beam characteristics for small field dosimetry using EGSnrc Monte Carlo code. Radiation Physics and Chemistry. 2017;135:63-75. doi: 10.1016/j.radphyschem.2017.02.029.

8. Lechner W, Palmans H, Solkner L, Grochowska
P, Georg D. Detector comparison for small field output factor measurements in flattening filter free photon beams. Radiother Oncol. 2013;109:35660. doi: 10.1016/j.radonc.2013.10.022. PubMed PMID: 24257020.

9. Lechner W, Wesolowska P, Azangwe G, Arib M, Alves VGL, Suming $L$, et al. A multinational audit of small field output factors calculated by treatment planning systems used in radiotherapy. Physics and Imaging in Radiation Oncology. 2018;5:58-63. doi: 10.1016/j.phro.2018.02.005.

10. Tyler MK, Liu PZ, Lee C, McKenzie DR, Suchowerska N. Small field detector correction factors: effects of the flattening filter for Elekta and Varian linear accelerators. J Appl Clin Med Phys. 2016;17:223-35. doi: 10.1120/jacmp.v17i3.6059. PubMed PMID: 27167280. PubMed PMCID: PMCPMC5690940.

11. Masanga W, Tangboonduangjit $P$, Khamfongkhruea C, Tannanonta C. Determination of small field output factors in 6 and $10 \mathrm{MV}$ flattening filter free photon beams using various detectors. Journal of Physics: Conference Series. 2016;698:012027. doi: 10.1088/1742-6596/694/1/012027.

12. Kragl G, Af Wetterstedt S, KnausI B, Lind M, McCavana $P$, Knoos T, et al. Dosimetric characteristics of 6 and 10MV unflattened photon beams. Radiother Oncol. 2009;93:141-6. doi: 10.1016/j. radonc.2009.06.008. PubMed PMID: 19592123.

13. IAEA. Commissioning and quality assurance of computerized planning systems for radiation treatment of cancer. Vienna: TRS Report Number 430; 2004. 